\title{
Fourier Transform Infrared Spectroscopy (FTIR) coupled with multivariate calibration and discriminant analysis for authentication of extra virgin olive oil from rambutan seed fat
}

\author{
${ }^{1}$ Nurwahidah, A.T., ${ }^{1}$ Rumiyati, ${ }^{1}$ Riyanto, S., ${ }^{2}$ Nurrulhidayah A.F., ${ }^{2}$ Betania K. and \\ $1,3^{*}$ Rohman, A. \\ ${ }^{1}$ Faculty of Pharmacy, Universitas Gadjah Mada, Yogyakarta 55281 Indonesia \\ ${ }^{2}$ International Institute of Halal Research and Training, International Islamic University Malaysia, 53100, \\ Gombak, Selangor, Malaysia \\ ${ }^{3}$ Institute of Halal Industry and Systems, Universitas Gadjah Mada, Yogyakarta 55281, Indonesia
}

\section{Article history:}

Received: 4 May 2019

Received in revised form: 7

June 2019

Accepted: 10 June 2019

Available Online: 11 June 2019

\section{Keywords:}

Olive oil,

Rambutan seed fat,

PCR,

Discriminant analysis,

Authentication

DOI:

https://doi.org/10.26656/fr.2017.3(6).182

\begin{abstract}
The adulteration practice in pharmaceutical industries, especially in fats and oils used as a vehicle in some pharmaceutical products must be identified to assure its quality. In this study, Fourier Transform Infrared Spectroscopy (FTIR) in combination with chemometrics techniques of multivariate calibration and discriminant analysis (DA) were used for the authentication of extra virgin olive oil (EVOO) from rambutan seed fat (RSF). EVOO, RSF, and the mixture of EVOO-RSF were scanned using FTIR spectrophotometer at mid-infrared region $\left(4000-650 \mathrm{~cm}^{-1}\right)$. The results showed that normal FTIR spectra at wavenumbers region of 1446.8-1409.7 $\mathrm{cm}^{-1}$ and 2368.6-1769.9 $\mathrm{cm}^{-1}$ combined with principle component regression (PCR) offered the best quantitative model for prediction of RSF levels in EVOO. The coefficient of determination $\left(\mathrm{R}^{2}\right)$ values obtained for the relationship between actual values of RSF and predicted values were of 0.9955 and 0.9915 in calibration and prediction models, respectively. The errors in calibration and prediction models were relatively low, accounting of $2.17 \%$ and $3.68 \%$, respectively. The classification model between unadulterated or pure EVOO and adulterated EVOO with RSF was successfully carried out using DA at wavenumbers of 3100-1000 $\mathrm{cm}^{-1}$ without any samples mistakenly classified into the wrong group. FTIR spectroscopy in combination with chemometrics offered effective tools for authentication of EVOO against the adulteration practice.
\end{abstract}

\section{Introduction}

Olive oil is one of the oil components used in some pharmaceutical preparations such as cream and lotion, therefore its authenticity is very important to assure the quality of pharmaceutical products. The adulteration practice of high-cost fats and oils with cheaper ones is common practice to be acted by unethical players in the fats and oils industry. For example, the substitution of olive oil with common vegetable oils like palm and corn oils can be very lucrative and attractive to get economical profits (Ferreiro-González et al., 2017). Extra virgin olive oil (EVOO), the highest grade of olive oil, command high price in the market due to its pleasant taste resulting from EVOO composition. The most common oils used as EVOO adulterants are seed oils including pomace, soy, sunflower, corn and rapeseed oils as well as nut oils, including peanut and hazelnut oils (Dankowska and Malecka, 2009). Rambutan seed fat
(RSF) is one of the seed fats potential adulterant in EVOO. RSF composed of $38 \%$ of rambutan seed (Manaf et al., 2013).

The diversity of oil adulterants on the market and the presence of adulteration practice have increased demand for analytical techniques needed to verify the authenticity of EVOO. Several analytical methods have been reported and validated for authentication analysis of EVOO. Most reported methods are relied on biological molecular techniques such as real-time polymerase chain reaction (Giménez et al., 2010; Alonso-Rebollo et al., 2017), chromatographic techniques, namely high performance liquid chromatography (Flores et al., 2006; Bajoub et al., 2017), gas chromatography using flame ionization detector (Jafari et al., 2009), gas chromatography in combination with atmospheric pressure chemical ionization-time of flight (GarcíaVillalba et al., 2011), differential scanning calorimetry 
(Van Wetten et al., 2015), and nuclear magnetic resonance spectroscopy (Dais and Hatzakis, 2013). These methods were time-consuming, needed sophisticated instruments and involved complex sample preparation, indeed, these methods were not preferred to be applied in routine monitoring systems. This challenged analytical chemists to develop some rapid and reliable techniques, especially based on spectroscopic methods such as UV-vis spectroscopy (Ferreiro-González et al., 2017) as well as fluorescence and Raman spectroscopy (Heise et al., 2005).

Combined with chemometrics (multivariate analysis), Fourier Transform Infrared Spectroscopy (FTIR) is an ideal technique for authentication of EVOO. FTIR spectroscopy offers a fast and reliable method, fingerprint in nature, and minimum or no sample preparation (Rohman and Che Man, 2012a). FTIR spectroscopy coupled with some chemometrics of principal component analysis (PCA), discriminant analysis, and multivariate calibrations has been reported for the authentication of EVOO from other vegetable oils of palm oil (Rohman and Che Man, 2010), sesame oil (Rohman and Che Man, 2012b), sunflower and corn oils (Özdemir and Öztürk, 2007), and hazelnut oil (Georgouli et al., 2017). FTIR spectroscopy was also used for the authentication of other edible oils such as cod liver oil (Rohman et al., 2017), butter (Nurrulhidayah et al., 2013), and red fruit oil (Larasati et al., 2013). In our best knowledge, there is no publication regarding the authentication of EVOO from rambutan seed fat (RSF). Therefore, the objective of this study was to develop FTIR spectroscopy in combination with chemometrics techniques for analysis of RSF as an adulterant in EVOO.

\section{Materials and methods}

\subsection{Materials}

Rambutan fruit was obtained from Yogyakarta, Indonesia and its authentication was performed in Department of Pharmaceutical Biology, Faculty of Pharmacy, Universitas Gadjah Mada, Yogyakarta. RSF was obtained by maceration of rambutan seed powder using methanol as an extracting solvent to get the methanolic extract. The methanol residue contained in the extract was evaporated using vacuum rotary evaporator. The extract was added with warm aquadest $\left(40^{\circ} \mathrm{C}\right)$ and then partitioned using hexane to get RSF. EVOO was purchased from the supermarket around Yogyakarta, Indonesia. The origin of EVOO used was from Spain. All chemicals and reagents used were of pro -analytical grade purchased from E. Merck (Darmstadt, Germany).

\subsection{Analysis of fatty acid composition}

Fatty acid composition of rambutan seed fat (RSF) and extra virgin olive oil (EVOO) was determined using gas chromatography (GC) equipped with flame ionization detector. GC condition and derivatization procedure were performed as in Che Man et al. (2011). Identification of each fatty acid methyl esters (FAMEs) was based on retention times of corresponding FAMEs in samples with those in FAMEs standards. Quantitative analysis of fatty acids was performed using relative percentage (internal normalization technique).

\subsection{Quantification of rambutan seed fat in EVOO using FTIR spectroscopy}

Authentication analysis of EVOO from RSF using FTIR spectroscopy was carried out by quantification of RSF in EVOO and classification of EVOO and EVOO mixed with RSF. Quantification of RSF in EVOO was facilitated by multivariate calibrations of partial least square (PLS) and principle component regression (PCR) by developing calibration and validation models. Preparation of calibration model was performed by making twenty samples containing EVOO mixed with RSF in certain concentration covering $1.0-50.0 \%(\mathrm{v} / \mathrm{v})$ RSF in EVOO. For model validation, 20 other independent samples were also made.

\subsection{Classification of EVOO and EVOO adulterated with $R S F$}

Classification of EVOO and that mixed with RSF was carried out using discriminant analysis (DA) by preparing EVOO from different brands and EVOO added with RSF at the concentration range of $1-50 \%$ of RSF. All EVOOs and EVOO mixed with RSF were assigned as "unadulterated" and "adulterated".

\subsection{FTIR spectra measurement}

FTIR spectra of studied oil samples were measured using FTIR spectrophotometer (Nicolet 6700 from Thermo Nicolet Corp., Madison, WI) equipped with detector of deuterated triglycine sulphate (DTGS) and beam splitter of $\mathrm{KBr} / \mathrm{Germanium}$. This instrument was interfaced to computer operating systems which include the OMNIC operating system (Version 7.0 Thermo Nicolet) software. The sampling compartment was horizontal Attenuated Total Reflectance kit (HATR, Smart ARK, Thermo Electron Corp.) composed of $\mathrm{ZnSe}$ crystal. All FTIR spectra of tested samples were scanned at wavenumbers of $4000-650 \mathrm{~cm}^{-1}$, using 32 scans with 4 $\mathrm{cm}^{-1}$ resolution. These spectra were recorded as absorbance mode at each data point in triplicate. 


\subsection{Data analyses}

The software of TQ Analyst ${ }^{\mathrm{TM}}$ version 6 (Thermo Electron Corporation, Madison, WI) was used during data analysis, including multivariate calibrations (PLS and PCR) and discriminant analysis (DA). The spectral regions where the variations were observed were chosen during analysis (PLS, PCR, and DA). For fatty acid analysis, the software of Excel ${ }^{\circledR}$ (Microsoft Corp., USA) was used for calculating the arithmetic mean and standard deviation (SD) of the mean.

\section{Results and discussion}

Fatty acid composition of extra virgin olive oil (EVOO) and rambutan seed fat (RSF) revealed that RSF contained palmitic acid $(5.50 \%)$, palmitoleic $(0.32 \%)$, stearic $(3.72 \%)$, oleic $(46.64 \%)$, linoleic $(3.92 \%)$, cis-11eicocinoic acid (27.39\%), eicosanoic (11.97\%) and erucic acid (0.54\%). While, the main fatty acid composed EVOO was palmitic acid (10.15\%), palmitoleic $(0.82 \%)$, stearic $(3.12 \%)$, oleic $(74.14 \%)$, linoleic (3.25\%), and linoleic acid (0.34\%). All fatty acids composed EVOO and RSF were in agreement specified in Codex Alimentarius, therefore, it can be concluded that the used EVOO and RSF were not adulterated previously.

Figure 1 shows the FTIR spectra of EVOO and RSF at wavenumbers of $4000-650 \mathrm{~cm}^{-1}$. Both spectra look similar due to its similarity in chemical contents in which the main components composed of EVOO and RSF are triacylglycerol (TAG). Each band and shoulders in FTIR spectra of EVOO and RSF corresponded to functional groups responsible for infrared absorption. Therefore, bands and shoulders of EVOO and RSF were attributed to functional groups present in TAG (Table 1). Due to its property as fingerprint in nature, both spectra (EVOO and RSF) could be differentiated by investigating the difference in band intensities, especially in band (1) due to stretching $=\mathrm{CH}$ vibration and in fingerprint region corresponding to bands at fingerprint region of 1500-650 $\mathrm{cm}^{-1}$. These bands were optimized for quantitative analysis and classification of RSF as an adulterant in EVOO. One of the reasons for selection of RSF as adulterant in EVOO was due to the similarity of RSF and EVOO in terms of the score of first principle component (PC1) and second principle component (PC2), obtained from principal component analysis (PCA), as shown in Figure 2. RSF and EVOO located in the same quadrant, meaning that FTIR spectra of RSF and EVOO were similar in variables used during PCA.

Quantitative analysis of RSF as adulterant in EVOO was facilitated by multivariate calibration of partial least square (PLS) and principle component regression (PCR). Both regressions are based on inverse calibration in which responses (concentration) in the y-axis and variables (absorbances) in the x-axis (Poulli et al., 2007). Selection of wavenumbers region is a critical point during the optimization of quantitative model. The wavenumbers region selected was based on the capability to provide the highest coefficient of determination $\left(\mathrm{R}^{2}\right)$ and the lowest errors in calibration (RMSEC) and in prediction (RMSEP). Therefore, wavenumbers which showed the difference between EVOO and RSF was selected. The software TQ Analyst automatically suggested wavenumbers region to be selected considering the FTIR difference of analytes (the combined wavenumbers region of $1446.8-1409.7 \mathrm{~cm}^{-1}$ and 2368.6-1769.9 $\mathrm{cm}^{-1}$ ). Some wavenumbers region was also evaluated, namely $3100-2900 \mathrm{~cm}^{-1}$ (clear band difference between EVOO and RSF), 1200-1000 $\mathrm{cm}^{-1}$

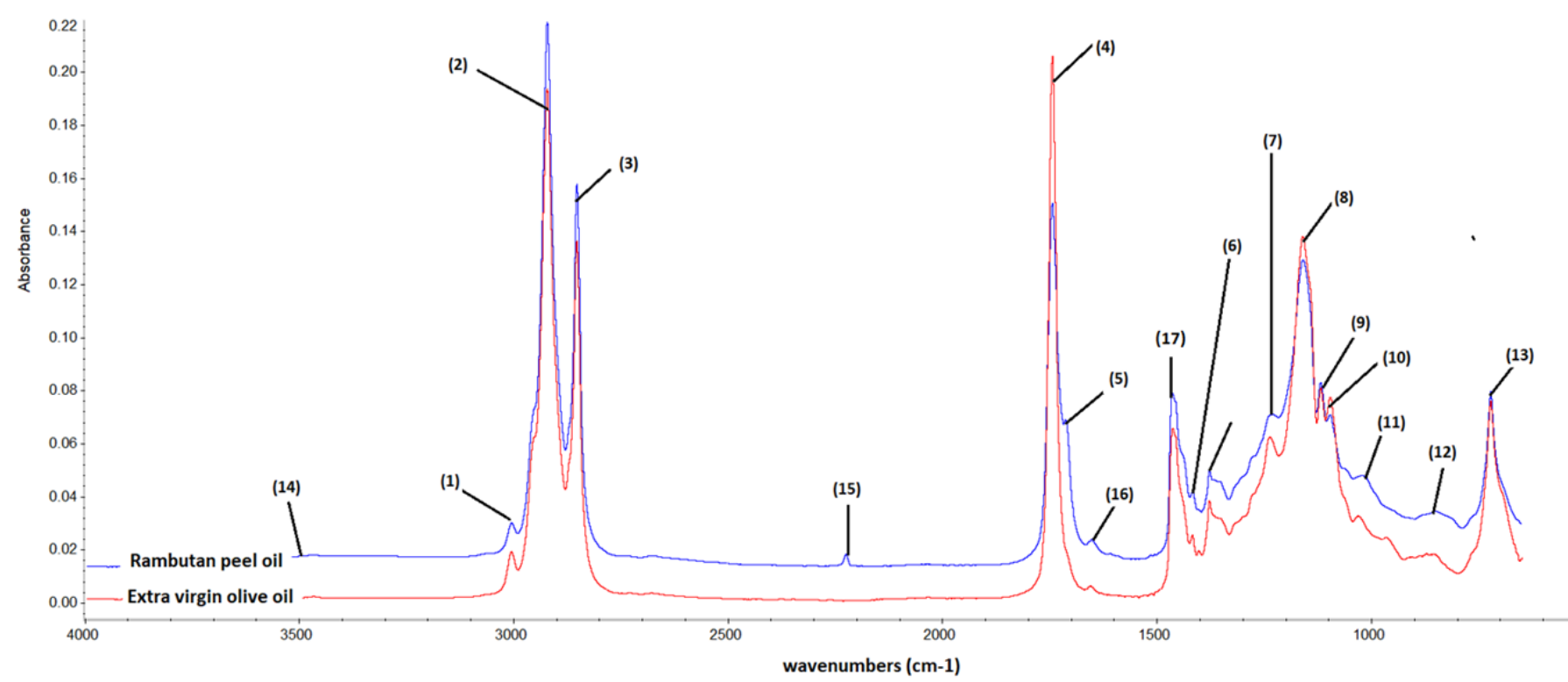

Figure 1. FTIR spectra of extra virgin olive oil (EVOO), grapeseed oil (GSO), soybean oil (SO), and walnut oil (WO) scanned at mid-infrared region $\left(4000-650 \mathrm{~cm}^{-1}\right)$. 
Table 1. Bands and shoulders corresponding to functional groups responsible for infrared absorption found in FTIR spectra of extra virgin olive oil (EVOO) and rambutan seed fat (Lerma-García et al., 2010).

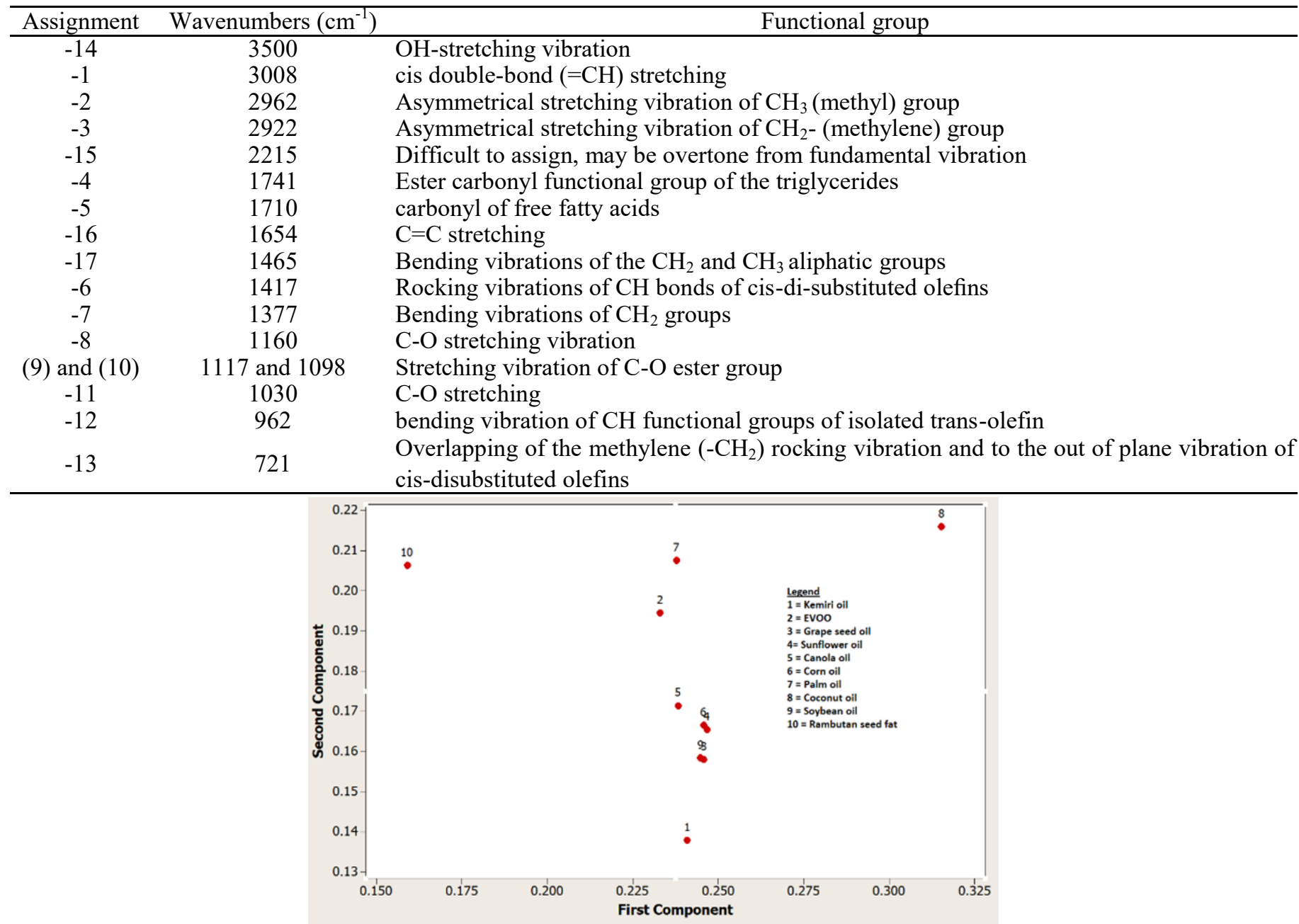

Figure 2. Principal component analysis of extra virgin olive oil (EVOO), rambutan seed fat (RSF) and other oils.

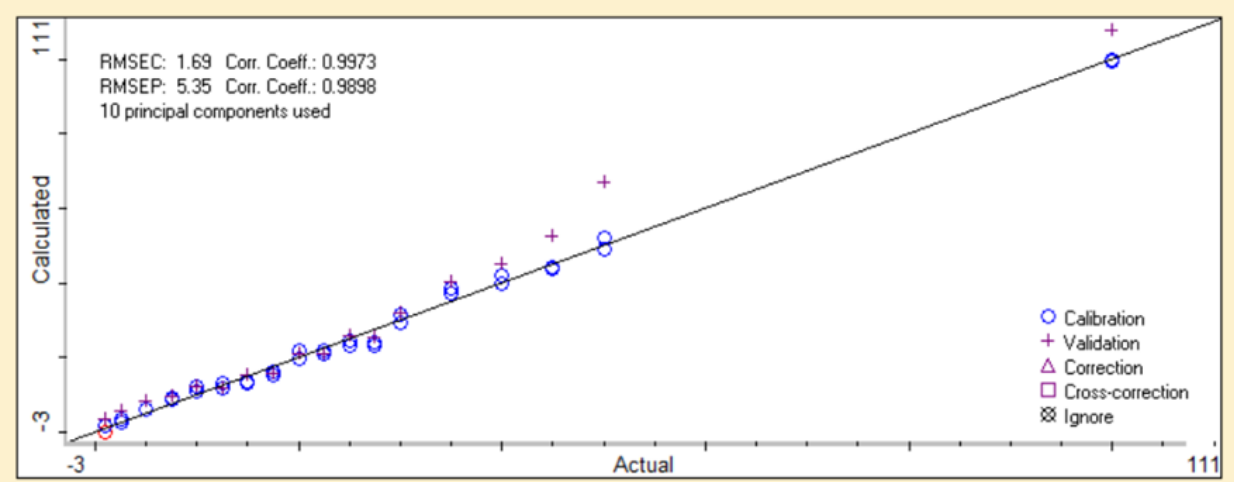

Figure 3. The principle component regression for the relationship between actual values of rambutan seed fat (RSF) and FTIR calculated values in calibration and validation models using the combined wavenumbers region of 1446.8-1409.7 and 2368.6$1769.9 \mathrm{~cm}^{-1}$.

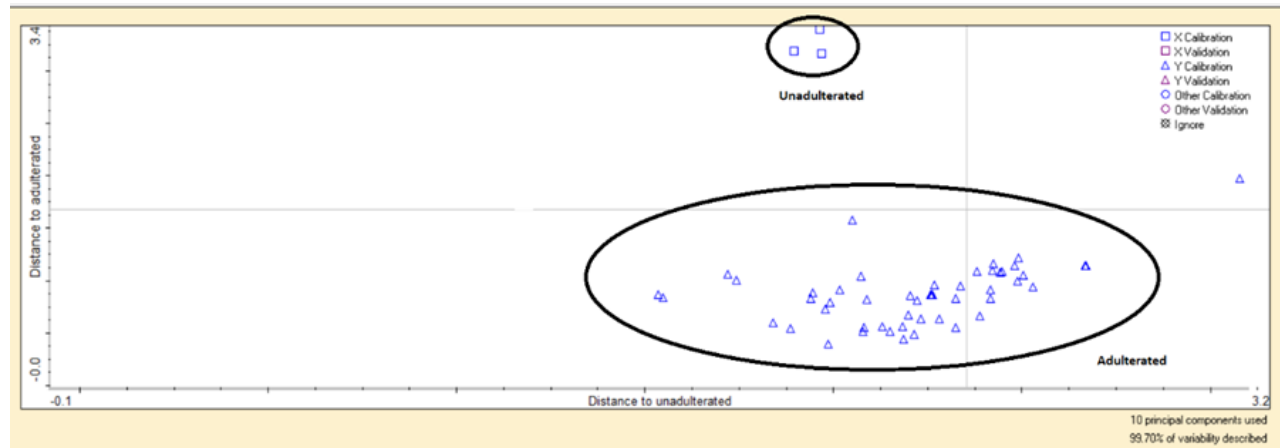

Figure 4. Discriminant analysis for classification of unadulterated extra virgin olive oil (EVOO) and adulterated EVOO with rambutan seed fat (RSF). 
due to fingerprint region revealing characteristic difference between EVOO and RSF, and its combination (combined wavenumbers region of $200-1000 \mathrm{~cm}^{-1}$ and $\left.3100-2900 \mathrm{~cm}^{-1}\right)$.

Table 2 tabulates the analytical results during quantification of RSF in EVOO. PCR using normal FTIR spectra at combined wavenumbers region of 1446.8-1409.7 and 2368.6-1769.9 $\mathrm{cm}^{-1}$ was selected for quantitative analysis of RSF in EVOO, offering the highest $\mathrm{R}^{2}$ (0.9955 in calibration model and 0.9915 in prediction model) and the lowest errors with RMSEC of $2.17 \%$ and RMSEP of $3.68 \%$. This result indicated that FTIR spectroscopy was accurate and precise for analysis of RSF in EVOO. Figure 3 reveals the calibration and validation models using PCR calibration. The equations obtained for the relationship between actual values of RSF ( $x$-axis) and FTIR predicted values (y-axis) were y $=1.0303 \mathrm{x}+1.3881$ (in calibration) and $\mathrm{y}=1.0516 \mathrm{x}+$ 1.2352 (validation). In addition, the residual analysis describing the difference between the actual value of RSF and predicted value exhibited scattered value above and below zero $(0)$. This indicated that systematic errors could be neglected, and the developed model was suitable for prediction of RSF in EVOO.

Classification of EVOO and EVOO adulterated with RSF using discriminant analysis (DA), a supervised pattern recognition technique. During DA, the wavenumbers regions were selected so that they offered distinct classification between EVOO and adulterated EVOO. Finally, the wavenumbers region of 3100-1000 $\mathrm{cm}^{-1}$ was selected during DA. Figure 4 exhibits the Coomans plot describing classification of EVOO and adulterated EVOO with RSF at concentration of $1-50 \%$ of RSF. DA could fruitfully classify "unadulterated EVOO" and "adulterated EVOO" with RSF with an accuracy level of $100 \%$, meaning that no samples were mistakenly classified into the wrong group.

\section{Conclusion}

Fourier Transform Infrared Spectroscopy (FTIR)in combination with the multivariate calibration of principle component regression (PCR) and discriminant analysis (DA) has been fruitfully used for quantification and classification of rambutan seed fat (RSF) in extra virgin olive oil (EVOO). The combined wavenumbers region of 1446.8-1409.7 and 2368.6-1769.9 $\mathrm{cm}^{-1}$ was selected over other regions for quantitative analysis of RSF in EVOO using PCR with acceptable accuracy (high $\mathrm{R}^{2}$ value) and precision (low RMSEC and RMSEP). DA was also successfully used for classification of EVOO and EVOO adulterated with RSF without any misclassification found.

\section{Conflict of Interest}

The author declared no conflict of interest.

\section{Acknowledgement}

The authors thank the Ministry of Research, Technology and Higher Education, Republic Indonesia for partial financial assistance during this study via Hibah Penelitian Unggulan Perguruan Tinggi (PUPT) 2018 with contract number: 185/UN1/DITLIT/DIT-LIT/ $\mathrm{LT} / 2018$.

Table 2. The performance of multivariate calibration for analysis of Rambutan seed fat as adulterant in extra virgin olive oil

\begin{tabular}{|c|c|c|c|c|c|c|c|}
\hline \multirow{2}{*}{$\begin{array}{l}\begin{array}{l}\text { Multivariate } \\
\text { calibration }\end{array} \\
\text { PLS }\end{array}$} & \multirow[t]{2}{*}{ Frequency region $\left(\mathrm{cm}^{-1}\right)$} & \multirow[t]{2}{*}{ Spectral treatment } & \multirow[t]{2}{*}{$\begin{array}{c}\text { Number of } \\
\text { factors }\end{array}$} & \multicolumn{2}{|c|}{ Calibration } & \multicolumn{2}{|c|}{ Validation } \\
\hline & & & & $\mathrm{R}^{2}$ & RMSEC & $\mathrm{R}^{2}$ & RMSEP \\
\hline & $1446.8-1409.7$ and $2368.6-1769.9$ & Normal & 4 & 0.9903 & 3.16 & 0.9862 & 4.66 \\
\hline & $1200-1000$ and $3100-2900$ & normal & 4 & 0.973 & 5.26 & 0.9744 & 5.33 \\
\hline & $1200-1000$ & normal & 1 & 0.9724 & 5.32 & 0.9751 & 5.14 \\
\hline & $3100-2900$ & normal & 2 & 0.9451 & 7.45 & 0.9474 & 7.82 \\
\hline & $1706-1663$ and $1780-1743$ & First derivative & & 0.9563 & 6.67 & 0.9815 & 5.33 \\
\hline & $1200-1000$ and $3100-2900$ & First derivative & & 0.9849 & 3.94 & 0.9858 & 5.13 \\
\hline & $1200-1000$ & First derivative & & 0.9889 & 3.39 & 0.9882 & 4.04 \\
\hline & $3100-2900$ & First derivative & 1 & 0.6908 & 16.5 & 0.8869 & 17.5 \\
\hline \multirow[t]{8}{*}{$\overline{\text { PCR }}$} & 1446.8-1409.7 and 2368.6-1769.9 & Normal & 10 & 0.9955 & 2.17 & 0.9915 & 3.68 \\
\hline & $1200-1000$ and $3100-2900$ & normal & 6 & 0.9881 & 3.51 & 0.9873 & 3.63 \\
\hline & $1200-1000$ & normal & 4 & 0.9858 & 3.83 & 0.9898 & 3.25 \\
\hline & $3100-2900$ & normal & 4 & 0.9852 & 3.9 & 0.9899 & 3.57 \\
\hline & $1706-1663$ and $1780-1743$ & First derivative & 10 & 0.9945 & 2.38 & 0.9935 & 5.15 \\
\hline & $1200-1000$ and $3100-2900$ & First derivative & 10 & 0.9973 & 1.69 & 0.9898 & 5.35 \\
\hline & $1200-1000$ & First derivative & 10 & 0.9964 & 1.92 & 0.9959 & 2.65 \\
\hline & $3100-2900$ & First derivative & 10 & 0.9958 & 2.1 & 0.9879 & 7.09 \\
\hline
\end{tabular}

The bold conditions were selected for prediction of RSF as adulterant in EVOO. 


\section{References}

Alonso-Rebollo, A., Ramos-Gómez, S., Busto, M.D. and Ortega, N. (2017). Development and optimization of an efficient qPCR system for olive authentication in edible oils. Food Chemistry, 232, 827-835. https:// doi.org/10.1016/j.foodchem.2017.04.078

Bajoub, A., Medina-Rodríguez, S., Gómez-Romero, M., Ajal, E., Bagur-González, M.G., FernándezGutiérrez, A. and Carrasco-Pancorbo, A. (2017). Assessing the varietal origin of extra-virgin olive oil using liquid chromatography fingerprints of phenolic compound, data fusion and chemometrics. Food Chemistry, 215, 245-255. https://doi.org/10.1016/ j.foodchem.2016.07.140

Che Man, Y.B., Rohman, A. and Mansor, T.S.T. (2011). Differentiation of lard from other edible oils by means of Fourier transform infrared spectroscopy and chemometrics. Journal of the American Oil Chemists' Society, 88(2), 187-192. https:// doi.org/10.1007/s11746-010-1659-x

Dais, P. and Hatzakis, E. (2013). Review Quality assessment and authentication of virgin olive oil by NMR spectroscopy: A critical review. Analytica Chimica Acta, 765, 1-27. https://doi.org/10.1016/ j.aca.2012.12.003

Dankowska, A. and Malecka, M. (2009). Application of synchronous fluorescence spectroscopy for determination of extra virgin olive oil adulteration. European Journal of Lipid Science and Technology, 111(12), 1233-1239. https://doi.org/10.1002/ ejlt.200800295

Ferreiro-González, M., Barbero, G.F., Álvarez, J.A., Ruiz, A., Ayuso, J. and Palma, M (2016). Authentication of virgin olive oil by a novel curve resolution approach combined with visible spectroscopy. Food Chemistry, 220, 331-336. https:// doi.org/10.1016/j.foodchem.2016.10.015

Flores, G., Ruiz Del Castillo, M.L., Herraiz, M. and Blanch, G.P. (2006). Study of the adulteration of olive oil with hazelnut oil by on-line coupled high performance liquid chromatographic and gas chromatographic analysis of filbertone. Food Chemistry, 97(4), 742-749. https://doi.org/10.1016/ j.foodchem.2005.06.008

García-Villalba, R., Pacchiarotta, T., Carrasco-Pancorbo, A., Segura-Carretero, A., Fernández-Gutiérrez, A., Deelder, A.M. and Mayboroda O.A. (2011). Gas chromatography-atmospheric pressure chemical ionization-time of flight mass spectrometry for profiling of phenolic compounds in extra virgin olive oil. Journal of Chromatography A, 1218(7), 959971. https://doi.org/10.1016/j.chroma.2010.12.014
Georgouli, K., Del Rincon, J.M. and Koidis, A. (2017). Continuous statistical modelling for rapid detection of adulteration of extra virgin olive oil using mid infrared and Raman spectroscopic data. Food Chemistry, 217, 735-742. https://doi.org/10.1016/ j.foodchem.2016.09.011

Giménez, M.J., Pistón, F., Martín, A.S. and Atienza, G. (2010). Application of real-time PCR on the development of molecular markers and to evaluate critical aspects for olive oil authentication. Food Chemistry, 118(2), 482-487. https://doi.org/10.1016/ j.foodchem.2009.05.012

Heise, H.M., Damm, U., Lampen, P., Davies, A.N. and Mcentry, P.S. (2005). Spectral variable selection for partial least squares calibration applied to authentication and quantification of extra virgin olive oils using Fourier transform Raman spectroscopy. Applied Spectroscopy, 59(10), 12861294. https://doi.org/10.1366/000370205774430927

Jafari, M., Kadivar, M. and Keramat J. (2009). Detection of adulteration in Iranian olive oils using instrumental (GC, NMR, DSC) methods. Journal of the American Oil Chemists' Society, 86(2), 103-110. https://doi.org/10.1007/s11746-008-1333-8

Larasati, D., Riyanto, S. and Rohman, A. (2013). Analysis of corn and soybean oils in red fruit oil using FTIR spectroscopy in combination with multivariate calibration. International Food Research Journal, 20, 1977-1981.

Lerma-García, M.J., Ramis-Ramos, G., HerreroMartínez, J.M. and Simó-Alfonso, E.F. (2010). Authentication of extra virgin olive oils by Fouriertransform infrared spectroscopy. Food Chemistry, 118(1), 78-83. https://doi.org/10.1016/ j.foodchem.2009.04.092

Manaf, Y.N.A., Marikkar, J.M.N., Long, K. and Ghazali, H.M. (2013). Physico-chemical characterization of the fat from red-skin rambutan (Nephelium lappaceum L.) seed. Journal of Oleo Science, 62(6), 335-343. https://doi.org/10.5650/jos.62.335

Nurrulhidayah, A.F., Che Man, Y.B., Rohman, A., Amin, I., Shuhaimi, M. and Khatib, A. (2013). Authentication analysis of butter from beef fat using FTIR spectroscopy coupled with chemometrics. International Food Research Journal, 20(4), 13831388.

Özdemir, D. and Öztürk, B. (2007). Near infrared spectroscopic determination of olive oil adulteration with sunflower and corn oil. Journal of Food Drug Analysis, 15, 40-47.

Poulli, K.I., Mousdis, G.A. and Georgiou, C.A. (2007). Rapid synchronous fluorescence method for virgin 
olive oil adulteration assessment. Food Chemistry, 105(1), 369-375. https://doi.org/10.1016/ j.foodchem.2006.12.021

Rohman, A. and Che Man, Y.B. (2012a). Application of Fourier Transform Infrared spectroscopy for Authentication of Functional Food Oils. Applied Spectroscopy Reviews, 47(1), 1-13. https:// doi.org/10.1080/05704928.2011.619020

Rohman, A. and Che Man, Y.B. (2012b). Authentication of extra virgin olive oil from sesame oil using FTIR spectroscopy and gas chromatography. International Journal of Food Properties, 15(6), 1309-1318. https://doi.org/10.1080/10942912.2010.521607

Rohman, A. and Che Man, Y.B. (2010). Fourier transform infrared (FTIR) spectroscopy for analysis of extra virgin olive oil adulterated with palm oil. Food Research International, 43(3), 886-892. https://doi.org/10.1016/j.foodres.2009.12.006

Rohman, A., Amalia, F. and Widyaningtyas, R. (2017). Authentication of cod liver oil from selected edible oils using FTIR spectrophotometry and chemometrics. International Food Research Journal, 24(4), 1362-1367.

Van Wetten, I.A., Van Herwaarden, A.W., BoerrigterEenling, S.R. and Van Ruth, S.M. (2015). Detection of sunflower oil in extra virgin olive oil by fast differential scanning calorimetry. Thermochimica Acta , 603, 237-243. https://doi.org/10.1016/ j.tca.2014.11.030 